\title{
Ação da L-glutamina via oral por 7 dias na estrutura e função muscular de pacientes com HIV/AIDS em uso de terapia antirretroviral
}

\author{
Action of L-glutamine orally for 7 days on structure and muscle function of HIVIAIDS patients \\ using antiretroviral therapy
}

DOI: 10.37111/braspenj.2020351008

Cervantes Caporossi

Camila da Silva Martins Ribas ${ }^{2}$

Diana Borges Dock-Nascimento ${ }^{3}$

Daniela de Brito Maurício ${ }^{4}$

\section{Unitermos:}

Glutamina. Sistema musculoesquelético. HIV. Sobreviventes de longo prazo ao HIV.

\section{Keywords:}

Glutamine. Musculoskeletal system. HIV. HIV longterm survivors.

\section{Endereço para correspondência:}

Cervantes Caporossi

Av. Fernando Corrêa da Costa, s/n - Coxipó - Cuiabá

MT, Brasil - CEP: 78000-000

E-mail: caporosi@terra.com.br

\section{Submissão}

17 de outubro de 2019

Aceito para publicação

6 de fevereiro de 2020

\begin{abstract}
RESUMO
Introdução: Pacientes infectados pelo vírus da imunodeficiência humana (HIV) têm sobrevida prolongada com o uso da terapia antirretroviral (TARV). A evolução crônica da doença permite aparecimento de alterações em vários sistemas, inclusive comprometimento nutricional e da função muscular. $O$ objetivo deste estudo é observar a ação da L-glutamina administrada via oral, na musculatura esquelética de pacientes com HIV/AIDS em uso crônico de TARV. Método: Pacientes soropositivos aderidos ao tratamento de TARV foram divididos em dois grupos: a) GG - grupo glutamina $(n=10)$ e b) $G M$ - grupo maltodextrina $(n=9)$. Os grupos foram suplementados, via oral e por 7 dias, sendo o GG com 0,5 mg/kg/dia de L-glutamina e o GM com 0,5 mg/kg/dia de maltodextrina. As variáveis de resultado analisadas foram: parâmetros de força muscular e quantidade de massa muscular, avaliadas por instrumentos de coleta, respectivamente, a dinamometria e a bioimpedância. Resultados: No GG, houve redução do peso corporal $(p=0,043)$ e aumento da força do bíceps $(p=0,006)$. No $G M$, houve aumento de peso corporal, acompanhado de ganho de massa muscular e perda de quantidade de gordura, sem provocar alterações significativas no índice de massa corporal. Conclusão: A L-glutamina, suplementada via oral, por 7 dias, aumentou a força muscular do bíceps de pacientes soropositivos em uso de TARV.
\end{abstract}

\section{ABSTRACT}

Introduction: Patients infected with the human immunodeficiency virus (HIV) have prolonged survival with the use of antiretroviral therapy (ART). The chronic evolution of the disease allows the appearance of changes in several systems, including nutritional impairment and muscular function. The objective of this study is to observe the action of orally administered L-glutamine on the skeletal muscles of patients with HIV/AIDS in chronic use of ART. Methods: Seropositive patients adhered to ART were divided into two groups: a) GG - glutamine group $(n=10)$ and $b)$ $G M$ - maltodextrin group ( $n=9)$. The groups were supplemented orally and for 7 days, $G G$ with $0.5 \mathrm{mg} / \mathrm{kg} /$ day of L-glutamine and GM with $0.5 \mathrm{mg} / \mathrm{kg} / \mathrm{day}$ of maltodextrin. The analyzed variables were: parameters of muscle strength and muscle mass, evaluated by collection instruments, respectively, dynamometry and bioimpedance. Results: There was a reduction in body weight $(p=0.043)$ and an increase in biceps strength $(p=0.006)$ in $G G$. In $G M$, there was an increase in body weight, accompanied by gain of muscle mass and loss of fat amount, without causing significant changes in body mass index. Conclusion: L-glutamine, orally supplemented, for 7 days, increased the muscle strength of the biceps of seropositive patients taking ART.

1. Doutorado em Medicina (Cirurgia do Aparelho Digestivo) pela Universidade de São Paulo; Professor Associado 4 da Universidade Federal de Mato Grosso; Orientador da Pós-graduação Universidade Federal de Mato Grosso; Universidade Federal de Mato Grosso, Departamento de Cirurgia, Cuiabá, MT, Brasil.

2. Mestranda do Programa de Pós-graduação em Ciências Aplicadas à Atenção Hospitalar; Enfermeira da Unidade de Bloco Cirúrgico, RPA e CME; Hospital Universitário Júlio Muller (HUJM), Cuiabá, MT, Brasil.

3. Doutorado em Ciências pela Universidade de São Paulo; Professora assistente 2 da Universidade Federal de Mato Grosso; Orientadora da Pós-graduação Universidade Federal de Mato Grosso, Cuiabá, MT, Brasil.

4. Mestre em Ciências da Saúde pela Faculdade de Medicina da Universidade Federal de Mato Grosso; Fisioterapeuta do Hospital Universitário Júlio Muller (HUJM), Cuiabá, MT, Brasil. 


\section{INTRODUÇ̃̃O}

Pacientes infectados pelo vírus da imunodeficiência humana (HIV) apresentam sobrevida prolongada com o uso da terapia antirretroviral (TARV), tornando a infecção pelo HIV uma condição clínica crônica. O tratamento possibilita o controle da carga viral e prolonga o tempo de vida dos pacientes, alterando o perfil histórico de alta mortalidade da AIDS para uma enfermidade crônica com complicações metabólicas e nutricionais'.

Estudos demonstram que a força muscular tem relação com a evolução da doença² e que a sua diminuição associada à sarcopenia (redução da massa muscular) contribui para o aumento da mortalidade ${ }^{3,4}$. A quantidade de massa muscular parece estar diretamente relacionada à função muscular, e a sua redução leva ao declínio da força muscular ${ }^{5}$. A perda de peso, particularmente de massa corporal magra, está relacionada com a diminuição da força muscular e do estado funcional, podendo levar ao aumento da mortalidade ${ }^{6}$.

Assim, a terapia nutricional (TN) é indicada com o objetivo de manter a composição corporal. Savy afirma que a suplementação do aminoácido glutamina reduz a perda de massa muscular esquelética, a diarreia e a má absorção, aumentando a defesa imune do hospedeiro e, consequentemente, melhorando a sua evolução ${ }^{7}$.

A glutamina é um componente essencial para numerosas funções metabólicas, tais como: homeostase ácido-base, gliconeogênese, transporte de nitrogênio, síntese de proteínas e ácidos nucléicos, assim como uma importante fonte de energia que tem sido usada como substrato energético suplementar. A suplementação com o referido aminoácido também tem sido usada em pacientes em pré e pós-operatórios e como restaurador de funções imunológicas em atletas de elite ${ }^{8,9}$.

Desta forma, a L-glutamina tem sido indicada no tratamento da sarcopenia, porém de forma muito limitada em pacientes com HIV. Assim sendo, consideramos interessante o estudo da glutamina como adjuvante no tratamento de pacientes com AIDS aderidos à TARV.

\section{MÉTODO}

Estudo prospectivo, ensaio clínico randomizado, realizado em pacientes portadores de HIV/AIDS e com acompanhamento ambulatorial especializado no município de Cuiabá - MT. O estudo foi previamente aprovado pelo Comitê de Ética e Pesquisa (CEP) da Universidade Federal de Mato Grosso (UFMT), de acordo com as diretrizes disciplinadas pela resolução 196/96 do Ministério da Saúde (Plataforma Brasil, número 042241/2012, parecer 255.111/2013). A coleta dos dados ocorreu no período de setembro de 2014 a maio de 2015 .
Foram incluídos no estudo pacientes de ambos os gêneros, com idades entre 18 e 60 anos, sorologia positiva para HIV, cadastrados na unidade e aderidos ao tratamento com TARV há 2 anos, bem nutridos (Avaliação Subjetiva Global - ASG A), sedentários e que aceitaram participar da pesquisa com assinatura prévia do Termo de Consentimento Livre e Esclarecido (TCLE).

Os critérios de exclusão foram: pacientes que iniciaram o tratamento há menos de 2 anos, amputação de algum dos membros, dificuldade de compreensão ou incapacidade de realizar o estudo, uso de qualquer tipo de nutriente e fármacos que interfiram nos resultados, como esteroides ou outra TARV, comorbidades metabólicas (diabetes, hepatopatias, doenças renais crônicas, desnutrição) e pacientes que não aceitaram participar do estudo ou não assinaram o TCLE.

Os voluntários foram randomizados em dois grupos: grupo glutamina ( $G G, n=10$, L-glutamina - 0,5 mg/kg/d) e grupo maltodextrina ( $G M, n=9$, maltodextrina $0,5 \mathrm{mg}$ / $\mathrm{kg} / \mathrm{d}$ ). A coleta dos dados foi realizada no primeiro dia (présuplementação) e no oitavo dia de intervenção. Todos os testes foram realizados no período da manhã e a avaliação foi feita unicamente pelo pesquisador responsável.

Para coleta dos valores de força muscular, tanto em membro inferior como superior, foi usado o dinamômetro MicroFet 2.

Para análise dos dados, foi utilizado método estatístico descritivo (média e desvio padrão). Por meio do teste de Shapiro Wilk, constatou-se a distribuição normal dos dados, então foi aplicado o test † Student nas amostras independentes, para verificar possíveis diferenças entre os grupos no momento inicial da coleta. A técnica de análise de variância (ANOVA) $2 \times 2$ para medidas repetidas foi utilizada para verificar diferenças nas variáveis nos grupos nos momentos analisados. A análise de covariância (ANCOVA) foi empregada na condição em que os grupos se apresentavam diferentes no momento inicial. As análises empregadas demonstraram com detalhes as alterações ocorridas nos efeitos do tempo, nos grupos e na interação entre eles. Feito teste de Fisher para pequenas proporções. critério de significância estatística estabelecido foi de $p<0,05$.

\section{RESULTADOS}

Foram selecionados 19 pacientes, conforme demonstrado no fluxograma de randomização (Figura 1).

A Tabela 1 demonstra semelhança entre os grupos conforme o sexo, a idade, a estatura, o peso, o índice de massa corporal (IMC), a gordura corporal, a massa muscular e a dinamometria do bíceps. 


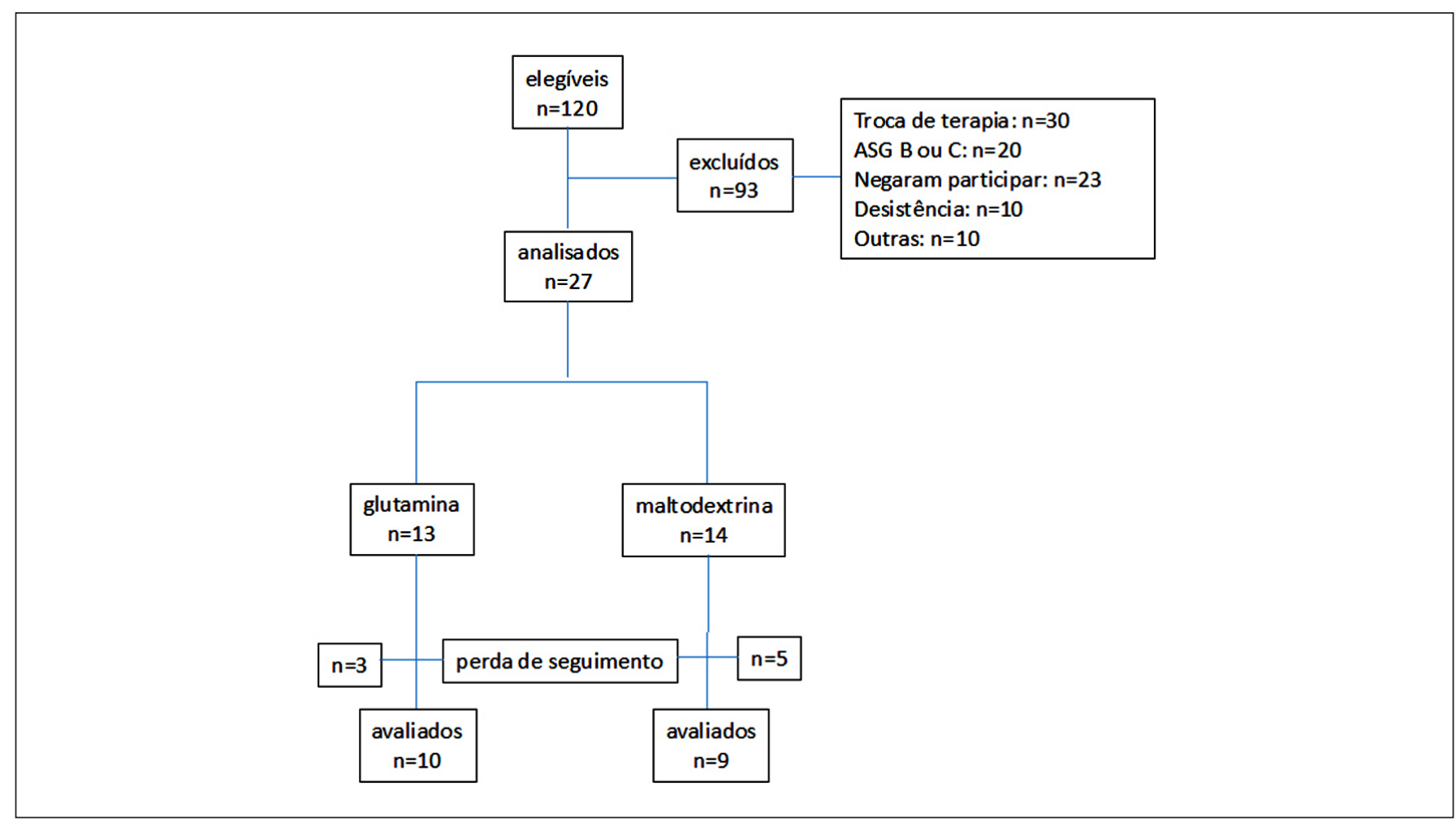

Figura 1 - Fluxograma de randomização.

Tabela 1 - Características da amostra.

\begin{tabular}{lccc}
\hline Variável & $\begin{array}{c}\text { GM } \\
\text { (Média, } \pm \text { DP) }\end{array}$ & $\begin{array}{c}\text { GG } \\
\text { (Média, } \pm \text { DP) }\end{array}$ & Valor de p \\
\hline Sexo (\%) & $78 \%+22 \% 0^{\lambda}$ & $40 \%+60 \% 0^{\lambda}$ & $0,115^{*}$ \\
Idade (anos) & $45 \pm 10,5$ & $37,6 \pm 6,41$ & 0,078 \\
Estatura (cm) & $1,68 \pm 0,06$ & $1,62 \pm 0,08$ & 0,065 \\
Peso (kg) & $76,33 \pm 15,11$ & $71,63 \pm 8,75$ & 0,412 \\
IMC (kg/m²) & $29,17 \pm 6,50$ & $25,11 \pm 4,21$ & 0,140 \\
Gordura (kg) & $26,11 \pm 12,74$ & $19,55 \pm 10,35$ & 0,233 \\
Massa muscular (kg) & $43,74 \pm 16,16$ & $38,40 \pm 13,86$ & 0,414 \\
Din. Bíceps (kgf) & $12,31 \pm 4,85$ & $15,48 \pm 5,92$ & 0,222 \\
Din. Quadríceps (kgf) & $15,91 \pm 4,93$ & $23,60 \pm 8,01$ & 0,024 \\
\hline
\end{tabular}

Din.= dinamometria; $\mathrm{GG}=$ grupo glutamina; $\mathrm{GM}=$ grupo malto; $\mathrm{IMC}=$ índice de massa corporal; kgf= kilograma força. Resultados expressos em valores médios $\pm \mathrm{DP}=$ desvio padrão. ${ }^{\star}$ Teste exato de Fisher.

Entretanto, quando da avaliação da dinamometria do quadríceps, os valores iniciais demonstram diferenças entre os grupos $(15,91 \pm 4,93 \mathrm{kgf}$ vs. $23,60 \pm 8,01 \mathrm{kgf}, \mathrm{p}=0,024)$.

A Tabela 2 revela valores das médias pré e pós-suplementação, segundo os grupos, e o valor de $p$, conforme o efeito em que foi analisado: tempo, grupo e grupo $x$ tempo (interação).

Os pesos no momento pré-suplementação foram semeIhantes entre os grupos, porém houve diferença após a suplementação na interação dos grupos. A média de peso do GM foi de 76,33 $\pm 15,11$ kg e, após a suplementação, de 77,08 $\pm 14,37 \mathrm{~kg}$. No GG, a média inicial foi de 71,63 $\pm 8,75 \mathrm{~kg}$ e, após suplementação, média de 71,32 \pm 8,72 kg. $O$ valor de $p=0,043$ (grupo $x$ tempo).

O IMC foi similar nos grupos. O GM apresentou no início média de 29,17 $\pm 6,50 \mathrm{~kg} / \mathrm{m}^{2}$ e, após a suplementação, de $29,37 \pm 6,26 \mathrm{~kg} / \mathrm{m}^{2}$. No $G G$, pré-suplementação, o grupo apresentou média de $25,11 \pm 4,21 \mathrm{~kg} / \mathrm{m}^{2}$, após, média de $25,45 \pm 4,17 \mathrm{~kg} / \mathrm{m}^{2}(p=0,712)$.

Os resultados foram semelhantes quanto à quantidade de gordura. $\bigcirc$ GM apresentou média pré-suplementação de $26,11 \pm 12,74 \mathrm{~kg}$ e, após suplementação, de 22,6 6 13,33 $\mathrm{kg}$. O GG inicialmente apresentou média de 19,55 $\pm 10,35$ $\mathrm{kg}$ e, após suplementação, de 19,76 $\pm 9,76 \mathrm{~kg}(p=0,103)$. 
Tabela 2 - Resultados das variáveis, pré e pós-suplementação, conforme os grupos.

\begin{tabular}{|c|c|c|c|c|}
\hline Variáveis & Grupo Maltodextrina & Grupo Glutamina & Valor de $p$ & Valor de $p$ \\
\hline Peso (kg) & & & ANOVA & \\
\hline Pré & $76,33 \pm 15,11$ & $71,63 \pm 8,75$ & Grupo & 0,353 \\
\hline Pós & & & Grupo x Tempo & $0,043^{*}$ \\
\hline IMC (kg/m²) & & & ANOVA & \\
\hline Pré & $29,17 \pm 6,50$ & $25,11 \pm 4,21$ & Grupo & 0,121 \\
\hline Pós & & & Grupo x Tempo & 0,712 \\
\hline Gordura (kg) & & & ANOVA & \\
\hline Pré & $26,11 \pm 12,74$ & $19,55 \pm 10,35$ & Grupo & 0,379 \\
\hline \multirow[t]{2}{*}{ Pós } & $22,60 \pm 13,33$ & $19,76 \pm 9,76$ & Tempo & 0,145 \\
\hline & & & Grupo x Tempo & 0,103 \\
\hline Pós & & & Grupo x Tempo & 0,085 \\
\hline Dinamometria Bíceps (kgf) & & & ANOVA & \\
\hline Pré & $12,31 \pm 4,85$ & $15,48 \pm 5,92$ & Grupo & 0,074 \\
\hline \multirow[t]{2}{*}{ Pós } & $10,23 \pm 4,21$ & $17,79 \pm 8,87$ & Tempo & 0,871 \\
\hline & & & Grupo x Tempo & $0,006^{*}$ \\
\hline Dinamometria Quadríceps (kgf) & & & ANOVA & \\
\hline Pré & $15,91 \pm 4,93$ & $23,60 \pm 8,01$ & Grupo & 0,317 \\
\hline \multirow[t]{2}{*}{ Pós } & $20,47 \pm 6,81$ & $24,57 \pm 9,42$ & Tempo & 0,040 \\
\hline & & & Grupo x Tempo & 0,167 \\
\hline
\end{tabular}

Médias e \pm DP= Desvio padrão. As análises foram feitas pela análise de variância (ANOVA), para a variável dinamometria do quadríceps foi utilizada análise de covariância (ANCOVA). * Interação grupo vs. tempo com $p<0,05$

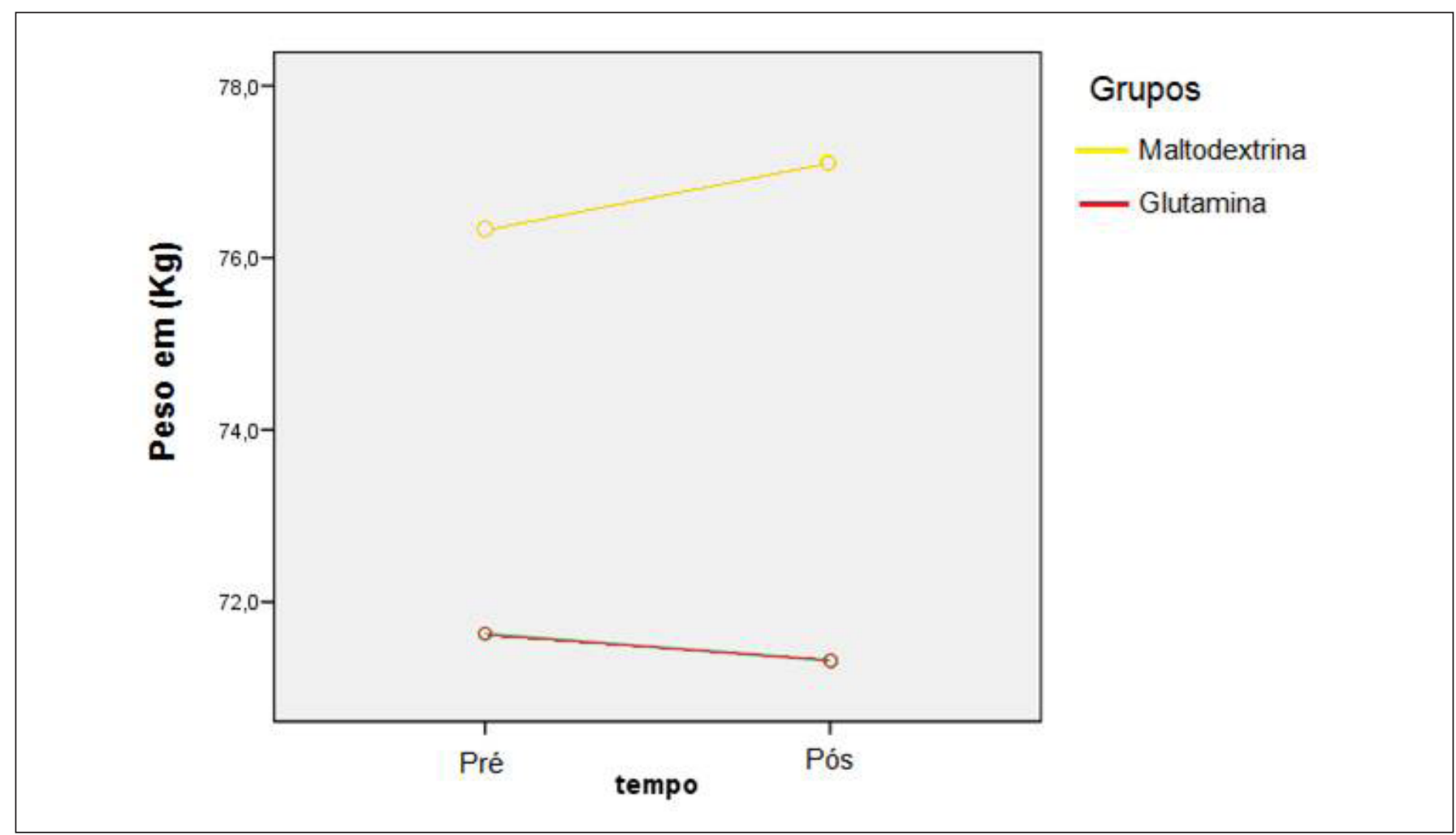

Figura 2 - Gráfico da variável peso, conforme os grupos, pré e pós-suplementação. 


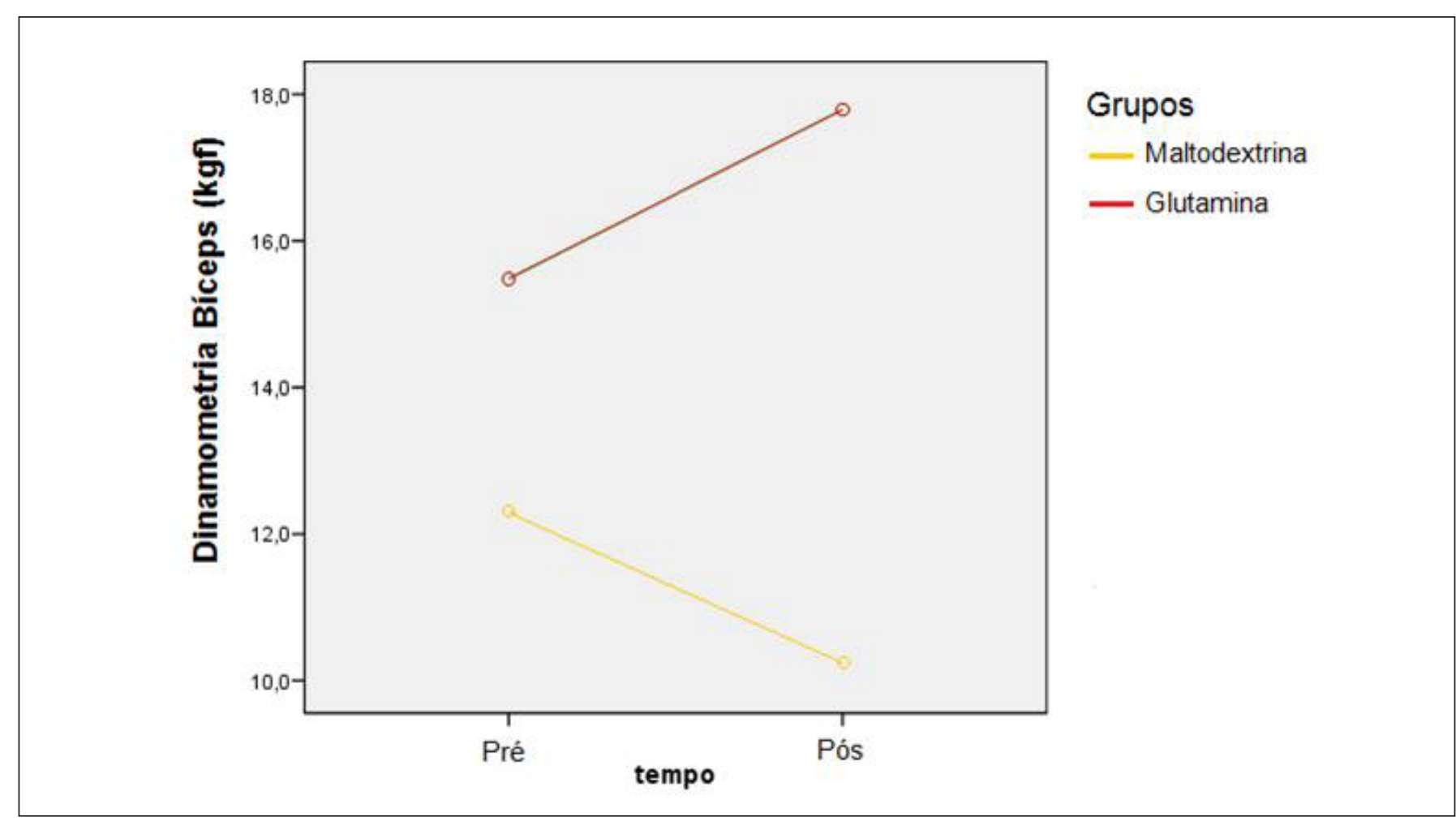

Figura 3 - Gráfico da variável dinamometria do biceps, conforme os grupos, pré e pós-suplementação.

Quanto à quantidade da massa muscular nos grupos, o GM pré-suplementação apresentou média de 43,74 \pm 16,16 $\mathrm{kg}$ e após, média de $51,7 \pm 10,98 \mathrm{~kg}$. No GG, a média pré-suplementação foi de $38,4 \pm 13,86 \mathrm{~kg}$ e após, 38,67 $\pm 14,86 \mathrm{~kg}(p=0,085)$.

Quanto à força do bíceps, houve diferença significativa nos grupos após a suplementação. No GM, a média de força do bíceps foi de 12,31 \pm 4,85 kgf pré suplementação, e de 10,23 $\pm 4,21$ kgf após. Já o GG apresentou antes do suplemento média de 15,48 $\pm 5,92$ kgf e, após suplementação, $17,79 \pm 8,87 \mathrm{kgf}$. O valor de $\mathrm{p}=0,006$ (grupo $\mathrm{x}$ tempo).

Quanto à dinamometria do quadríceps, o $\mathrm{GM}$ apresentou média de força pré-suplementação de 15,91 \pm 4,93 kgf, e após, 20,47 $\pm 6,81 \mathrm{kgf}$. O GG, inicialmente, teve média de $23,6 \pm 8,01$ kgf e, após suplementação, de 24,57 \pm 9,42 kgf. Não houve diferença significativa na interação entre eles $(p=0,167$ - grupo $x$ tempo), mas no efeito isolado do tempo houve diferença, demonstrando aumento da média de força do músculo quadríceps no tempo, independente do grupo, todos tiveram aumento $(p=0,040$ - tempo).

\section{DISCUSSÃO}

A ação da L-glutamina na musculatura esquelética em soropositivos durante TARV foi avaliada no estudo, demonstrando aumento da força muscular do bíceps. A comparação dos nossos resultados com o de outros autores fica dificultado, visto que os estudos da L-glutamina em pacientes HIV/AIDS são escassos. Somado a isto, há muita variação nos tipos de suplementos associados, no perfil dos pacientes e nas variadas drogas utilizadas como antirretroviral.

O IMC está fortemente associado à incidência de mortalidade desses pacientes ${ }^{10}$, por isso, para melhor compreensão acerca das alterações corporais provocadas pelo HIV, somado aos efeitos colaterais da TARV, realizamos a análise da composição corporal para obter maiores detalhes dessas alterações.

Nos anos iniciais da epidemia da AIDS, havia o estigma da perda de peso significativa nos indivíduos infectados. Atualmente, o cenário mudou e, assim como no nosso estudo, outros relatos demonstram que o peso encontrado é normal ou até mesmo sobrepeso $2,11,12$.

Clark et al. ${ }^{13}$, com grupos suplementados com maltodextrina e L-glutamina, descreveram ganho de peso corporal em ambos os grupos. Entretanto, nossa pesquisa relevou que $\circ$ ganho de peso foi identificado somente no $\mathrm{GM}$, enquanto, no $G G$, foi observada perda $(p=0,043)$. Deve ser considerado o fato que, no estudo citado, a L-glutamina estava associada à arginina e ao hidroximetilbutirato, além do tempo maior de suplementação ${ }^{13}$.

Estudo realizado em um grupo de homens jovens hígidos suplementados antes e após exercício excêntrico demonstrou que a suplementação oral aguda também associada de 
leucina e leucina + glutamina aumentou a taxa de recuperação na força isométrica, altura do salto contra-movimento, dor muscular de início tardio e creatina quinase comparado ao placebo ${ }^{14}$. Alterações na composição corporal de soropositivos podem ou não alterar o IMC, mesmo considerando ganho ou perda de peso corporal ${ }^{15}$. Assim como no IMC, nas variáveis quantidade de gordura e de massa muscular, não ocorreram diferenças entre os grupos suplementados.

Considerando que a função muscular pode sofrer alterações com o estado nutricional, a dinamometria mensura a força do músculo esquelético, e pode demonstrar modificações funcionais antes de surgirem diferenças morfológicas clinicamente detectáveis ${ }^{16}$. Pode ser utilizada como referência para avaliar o risco de mortalidade ${ }^{2}$.

A força do músculo bíceps apresentou aumento significativo no $G G(p<0,006)$. Waddell et al. ${ }^{17}$ avaliaram o efeito da glutamina na força de contração muscular esquelética de rato, e observaram ganho de massa muscular e da força de contração. Rahmani-Nia et al. ${ }^{18}$, em estudo realizado em humanos, concluíram que resultados favoráveis ao aumento da força podem estar relacionados à otimização da síntese proteica devido à oferta da L-glutamina. Freitas ${ }^{19}$, em pesquisa com voluntários não soropositivos, após administrar L-glutamina, concluiu que a suplementação resultou em evidente aumento de força muscular no membro superior. Holecek ${ }^{20}$, ofertando L-glutamina, concluiu que a sua suplementação é fundamental para a manutenção do seu nível em estados de catabolismo, além de atuar como precursor para síntese de proteínas musculares, glucose e ácidos nucleicos.

Quanto ao músculo quadríceps, inicialmente, o GG apresentou médias de força superiores comparado ao $G M$, após o experimento, a análise de covariância demonstrou diferença significativa apenas no efeito isolado do tempo, e não na interação entre os grupos (grupo x tempo), ou seja, não houve diferença significativa, como também foi relatado por Rahmani-Nia et al. ${ }^{21}$, em indivíduos não infectados pelo HIV, ao comparar grupos após suplementação com maltodextrina e gln.

Em contrapartida, estudo realizado, utilizando suplementação de L-glutamina + maltodextrina e esta separadamente como placebo, com indivíduos saudáveis para examinar a força muscular do quadríceps e classificação da dor pós exercício, resultou em maior pico de torque e menores taxas de dor pós exercício com a L-glutamina, especialmente em homens ${ }^{22}$.

Ensaio clínico randomizado em recrutamento no Japão com pacientes de idade maior ou igual a 65 anos em préoperatório de cirurgia cardíaca mostrará se a pré-suplementação associada de leucina, glutamina e arginina irá apresentar resultados satisfatórios quanto à força muscular e composição corporal, dentre outros desfechos relacionados à suplementação $0^{23}$.
Na presente pesquisa, o efeito da suplementação com L-glutamina na força do músculo bíceps foi diferente do músculo quadríceps. Esta diferença poderia ser justificada considerando que, o bíceps é um músculo com predomínio de fibras musculares do tipo l, oxidativas (40\%), que podem apresentar três vezes mais estoque de glutamina quando comparadas às fibras do tipo II, glicolíticas ${ }^{24,25}$. Assim, a suplementação com a glutamina determina melhor desempenho de força nesse grupo muscular quando avaliado comparativamente ao quadríceps.

Considerando, também, que estudos que avaliaram quantidades de massa e de força muscular, relatam que a musculatura esquelética se comporta de maneiras variáveis, conforme o diagnóstico da doença e a atividade de vida diária do paciente ${ }^{26-28}$. Para Ruiter et al. ${ }^{27}$, em algumas situações, a função muscular pode ser afetada de diferentes formas, comprometendo mais os membros superiores ou inferiores, com intensidade também variável.

O comprometimento nutricional desses pacientes tem sido estudado atualmente como um determinante no aumento da suscetibilidade de infecções oportunistas. Na medida em que aumenta a expectativa de vida, nos deparamos com a instalação de outras enfermidades que, a longo prazo, causam impacto na qualidade de vida destes pacientes. É importante que os profissionais de saúde que cuidam de indivíduos infectados pelo HIV compreendam a relação entre a nutrição, a infecção pelo HIV e a musculatura esquelética.

As limitações do nosso estudo, como o tamanho da amostra, seleção de pacientes sem risco nutricional, o tempo de suplementação da L-glutamina e a dose ofertada, sugerem a necessidade de realização de novas pesquisas acerca do assunto. Estudos recentes com associações de aminoácidos têm mostrado resultados satisfatórios, porém, em virtude da fusão de compostos, ficamos limitados em discutir o efeito isolado da glutamina na resposta muscular dos indivíduos.

\section{CONCLUSÃO}

A suplementação da L-glutamina, por 7 dias, via oral, aumentou a força muscular do bíceps de pacientes com HIV/AIDS.

\section{REFERÊNCIAS}

1. Srinivasa S, Grinspoon SK. Metabolic and body composition effects of newer antiretrovirals in HIV-infected patients. Eur J Endocrinol. 2014;170(5):R185-202.

2. Newman AB, Kupelian V, Visser M, Simonsick EM, Goodpaster $\mathrm{BH}$, Krirchevsky SB, et al. Strength, but not muscle mass, is associated with mortality in the health, aging and body composition study cohort. J Gerontol A Biol Sci Med Sci. 2006;61(1):72-7.

3. Metter EJ, Talbot LA, Schrager M, Conwit R. Skeletal muscle strength as a predictor of all-cause mortality in healthy men. J Gerontol A Biol Sci Med Sci. 2002;57(10):B359-65. 
4. Rantanem T, Harris T, Leveille SG, Visser M, Foley D, Masaki $\mathrm{K}$, et al. Muscle strength and body mass index as long-term predictors of mortality in initially healthy men. J Gerontol A Biol Sci Med Sci. 2000;55(3):M168-73.

5. FronteraWR,MeredithCN,O'ReillyKP,KnuttgenHG,Evans WJ. Strength conditioning in older men: skeletal muscle hypertrophy and improved function. J Appl Physiol. 1988;64(3):1038-44.

6. SociedadeBrasileira de Nutrição Parenteral e Enteral,Associação Brasileira de Nutrologia. Projeto diretrizes. Terapia nutricional na síndrome da imunodeficiência adquirida (HIV/AIDS). São Paulo: Associação Médica Brasileira e Conselho Federal de Medicina; 2011. p.1-12.

7. Savy GK. Glutamine supplementation: heal the gut, help the patient. J Infus Nurs. 2002;25(1):65-9.

8. Oliveira GP, Dias CM, Pelosi P, Rocco PR. Understanding the mechanisms of glutamine action in critically ill patients. An Acad Bras Ciênc. 2010;82(2):417-30.

9. Cruzat V, Rogero MM, Keane KN, Curi R, Newsholme P. Glutamine: metabolism and immune function, supplementation and clinical translation. Nutrients. 2018;10(11). pii: E1564.

10. Pee S, Semba RD. Role of nutrition in HIV infection: review of evidence for more effective programming in resource-limited settings. Food Nutr Bull. 2010;31(4 suppl):S313-44.

11. Evans D, McNamara L, Maskew M, Selibas K, van Amsterdam $\mathrm{D}$, Baines $\mathrm{N}$, et al. Impact of nutritional supplementation on immune response, body mass index and bioelectrical impedance in HIV-positive patients starting antiretroviral therapy. Nutr J. 2013; 12:111.

12. Shabert JK, Winslow C, Lacey JM, Wilmore DW. Glutamineantioxidant supplementation increases body cell mass in AIDS patients with weight loss: a randomized, double-blind controlled trial. Nutrition. 1999;15(11-12):860-4.

13. Clark RH, Feleke G, Din M, Yasmin T, Singh G, Khan FA, et al. Nutritional treatment for acquired immunodeficiency virus-associated wasting using $\beta$-hydroxy $\beta$-methylbutyrate, glutamine, and arginine: a randomized, double-blind, placebo-controlled study. JPEN J Parenter Enteral Nutr. 2000;24(3):133-9.

14. Waldron M, Ralph C, Jeffries O, Tallent J, Theis N, Patterson SD. The effects of acute leucine or leucine-glutamine co-ingestion on recovery from eccentrically biased exercise. Amino Acids. 2018;50(7):831-9.

15. Denue BA, Ikunaiye PN, Denue CB. Body mass index changes during highly active antiretroviral therapy in Nigeria. East Mediterr Health J. 2014;19(suppl. 3):S89-97.

16. Wilson IB, Roubenoff R, Knox TA, Spiegelman, Gorbach SL. Relation of lean body mass to health-related quality of life in persons with HIV. J Acquir Immune Defic Syndr. 2000;24(2):137-46.

17. Waddell D, Fredricks K. Effects of a glutamine supplement on the skeletal muscle contractile force of mice. Am J Undergraduate Research. 2005;4(2):11-8.

18. Rahmani-Nia F, Farzaneh E, Damirchi A, Majlan AS, Tadibi V. Surface electromyography assessments of the vastus medialis and rectus femoris muscles and creatine kinase after eccentric contraction following glutamine supplementation. Asian J Sports Med. 2014;5(1):54-62.

19. Freitas AMP. Efeito da suplementação com glutamina sobre a inflamação sistêmica e a composição corporal de idosos [Dissertação de mestrado]. São Paulo: Universidade de São Paulo; Mestrado em Interunidades Nutrição Humana Aplicada - PRONUT; 2014. 148p.

20. Holecek M. Side effects oflong-term glutamine supplementation. JPEN J Parenter Enteral Nutr. 2013;37(5):607-16.

21. Rahmani Nia F, Farzaneh E, Damirchi A, Shamsi Majlan A. Effect of L-glutamine supplementation on electromyographic activity of the quadriceps muscle injured by eccentric exercise. Iran J Basic Med Sci. 2013;16(6):808-12.

22. Legault Z, Bagnall N, Kimmerly DS. The influence of oral L-glutamine supplementation on muscle strength recovery and soreness following unilateral knee extension eccentric exercise. Int J Sport Nutr Exerc Metab. 2015;25(5):417-26.

23. Ogawa M, Yoshida N, Satomi-Kobayashi S, Tsuboi Y, Komaki $\mathrm{K}$, Wakida K, et al. Efficacy of preoperative amino acid supplements on postoperative physical function and complications in open heart surgery patients: a study protocol for a randomized controlled trial. J Cardiol. 2019;74(4):360-5.

24. Eriksson PO. Muscle-fibre composition of the human mandibular locomotor system. Enzyme-histochemical and morphological characteristics of functionally different parts. Swed Dent J Suppl. 1982;12(Suppl):1-44.

25. Cruzat VF, Petry ER, Tirapegui J. Glutamina: aspectos bioquímicos, metabólicos, moleculares e suplementação. Rev Bras Med Esporte. 2009;15(5):392-7.

26. Man WD-C, Soliman MGG, Nikoletou D, Harris ML, Rafferty GF, Mustfa N, et al. Non-volitional assessment of skeletal muscle strength in patients with chronic obstructive pulmonary disease. Thorax. 2003;58(8):665-9.

27. Ruiter CJ, Jongen PJ, van der Woude LH, de Haan A. Contractile speed and fatigue of adductor pollicis muscle in multiple sclerosis. Muscle Nerve. 2001;24(9):1173-80.

28. Edwards RH, Young A, Hosking GP, Jones DA. Human skeletal muscle function: description of tests and normal values. Clin Sci Mol Med. 1977;52(3):283-90.

Local de realização do estudo: Universidade Federal de Mato Grosso, Cuiabá, MT, Brasil.

Conflito de interesse: Os autores declaram não haver. 